\title{
Epidemiologia das fraturas toracolombares cirúrgicas na zona leste de São Paulo
}

\author{
Epidemiology of thoracolumbar surgical fractures in the east side of \\ São Paulo
}

\author{
Epidemiología de las fracturas toracolumbares quirúrgicas en la \\ región Leste de São Paulo
}

\author{
Luiz Cláudio Lacerda Rodrigues ${ }^{1}$ \\ Adalberto Bortoletto ${ }^{2}$ \\ Marcelo Hide Matsumoto ${ }^{3}$
}

\section{RESUMO}

Objetivo: fazer uma análise prospectiva de cem pacientes com fratura da coluna toracolombar submetidos a tratamento cirúrgico em um hospital terciário da zona leste de São Paulo. Métodos: estudo prospectivo com início em Janeiro de 2006 até Julho de 2009, sendo incluído um total de 100 pacientes com fratura da coluna toracolombar submetidos a tratamento cirúrgico. Avaliou-se o sexo, a idade, o mecanismo de trauma, o déficit neurológico e o tipo de fratura. Resultados: foi observado que queda de altura foi o principal fator causador deste tipo de fratura seguido de acidentes com veículos automotores. O sexo masculino foi responsável por $66 \%$ dos casos, porém não foi observada diferença entre o mecanismo de trauma e o sexo. Observamos também que a lesão neurológica apresenta relação direta com a gravidade de fratura e que a junção toracolombar é a região mais acometida. Conclusão: concluímos que a incidência de

\section{ABSTRACT}

Objective: to carry out a prospective analysis of 100 patients with thoracolumbar spine fractures who underwent surgery at a tertiary hospital in the east side of São Paulo, Brazil. Methods: a prospective study from January 2006 until July 2009, including an overall of 100 patients with fractures of the thoracolumbar spine who underwent surgery. Data such as gender, age, mechanism of injury, neurologic deficit and type of fracture were assessed. Results: it was observed that the fall of slab was the main cause of fracture, followed by car accidents, and males accounted for $66 \%$ of the cases; however, no difference was observed between the mechanism of trauma and the gender. We also observed that neurological damage is directly related to severity of fracture and that the thoracolumbar junction is the most affected region. Conclusion: we conclude that the incidence of fractures in the east side of São

\section{RESUMEN}

Objetivo: hacer un análisis prospectivo de cien pacientes con fracturas de la columna toracolumbar sometidos al tratamiento quirúrgico en un hospital de tercer nivel de la región Leste de la ciudad de São Paulo. Métodos: estudio prospectivo con inicio en Enero de 2006 hasta Julio de 2009, donde fueron incluidos un total de cien pacientes con fractura de la columna toracolumbar sometidos al tratamiento quirúrgico. Se evaluaron el sexo, la edad, el mecanismo del trauma, el déficit neurológico y el tipo de fractura. Resultados: fue observado que la caída de una altura fue el principal factor causante de este tipo de fractura, seguido de accidentes con vehículos automotores. El sexo masculino fue responsable por el $66 \%$ de los casos, sin embargo no fue observada una diferencia entre el mecanismo del trauma y el sexo. Se observó también que la lesión neurológica presenta relación directa con la gravedad de la fractura y que la

\footnotetext{
Setor de Ortopedia e Traumatologia do Hospital Santa Marcelina - São Paulo (SP), Brasil.

' Médico assistente do Serviço de Patologias da Coluna Vertebral do Hospital Santa Marcelina - São Paulo (SP), Brasil.

${ }^{2}$ Médico chefe do Serviço de Patologias da Coluna Vertebral do Hospital Santa Marcelina - São Paulo (SP), Brasil.

${ }^{3}$ Chefe do Serviço de Ortopedia e Traumatologia do Hospital Santa Marcelina - São Paulo (SP), Brasil.

Não há conflitos de interesse.

Não houve fonte pagadora ou patrocínio para realização deste trabalho. 
fraturas na zona leste de São Paulo é elevada e associam-se a quedas acidentais. Verifica-se que estes dados são importantes para que medidas de prevenção possam ser tomadas com vistas a diminuir a morbidade deste grave tipo de trauma.
Paulo and it is highly associated with accidental falls. These data are important so that preventive measures can be undertaken to reduce the morbidity of this type of severe trauma.

junción toracolumbar es la región más comprometida. Conclusión: la incidencia de fracturas en la región Leste de São Paulo es elevada y asociada con caídas accidentales. Fue observado que estos datos son importantes para que medidas de prevención puedan ser realizadas, de forma que disminuya la morbilidad de este tipo de trauma grave.

KEYWORDS: Spinal injuries/ epidemiology; Spinal injuries/ surgery
DESCRIPTORES: Traumatismos vertebrales/epidemiología; Traumatismos vertebrales/ cirugía epidemiologia; Traumatismos da coluna vertebral/cirurgia

\section{INTRODUÇÃO}

A Zona Leste de São Paulo é a área situada a leste do Rio Tamanduateí, com uma população de 3.620.494, segundo dados da prefeitura ${ }^{1}$.

Fraturas da coluna toracolombar impactam na vida do paciente e de sua família. Acarretam um elevado custo ao sistema de saúde pública, incluindo: internações, cirurgias e reabilitação associadas à perda da produtividade do paciente, algumas vezes definitivamente ${ }^{2}$.

Estudos baseados em análises epidemiológicas servem para informar uma estimativa local sobre prevalência e grupos de risco, e para fornecer dados para que um trabalho de prevenção possa ser feito.

Este estudo prospectivo com início em Janeiro de 2006 teve por objetivo mostrar a estatística dos pacientes submetidos ao tratamento cirúrgico, assim como descrever a população em risco e incrementar um trabalho de prevenção.

\section{MÉTODOS}

Foram incluídos no estudo todos os pacientes com fraturas nos segmentos torácico e lombar que preencheram critérios de instabilidade baseados na classificação $\mathrm{AO}^{3}$ e que apresentaram condições clínicas passíveis de submissão a tratamento cirúrgico no Hospital Santa Marcelina, entre o período de Janeiro de 2006 a Agosto de 2009.

Foram excluídas do estudo fraturas por osteoporose, fraturas patológicas, fraturas do segmento cervical, fraturas crônicas com mais de 30 dias de evolução e fraturas submetidas a tratamento conservador.

Foram coletadas informações referentes à idade, sexo, mecanismo de trauma, tipo de fratura, status neurológico e nível da lesão. A classificação do tipo de fratura foi baseada na classificação $\mathrm{AO}^{3}$, e o déficit neurológico foi avaliado pela Escala de Frankel ${ }^{4}$.

Os dados descritivos foram analisados por meio de proporções e médias, sendo os dados avaliados individualmente e as relações importantes, cruzadas e examinadas, e os indicadores serão apresentados.

\section{RESULTADOS}

\section{Epidemiologia}

Foram avaliados 100 pacientes com uma média de 30 novos casos por ano. A relação entre homens e mulheres é 2:1 (teste do $\chi^{2}$ de aderência com $p=0,001$ ) (Figura 1). A média de idade foi 38 anos, variando de 16 a 67 com um desvio padrão de 12,6 (Figura 2). Não foi observada variação dentro da faixa etária. Todos os pacientes da pesquisa eram economicamente ativos.

\section{Etiologia}

A causa mais comum de fratura foi queda de altura, com um total de 78 casos, dentre os quais 75 devido à queda de lajes, 2 à queda de árvores, 1 à queda de janela de edifício ( $3^{\circ}$ andar) (Figura 3), e 22 devido a acidentes com veículos automotores, sendo observados 12 acidentes com moto, 9 com carro e 1 atropelamento. O número de quedas é signi-

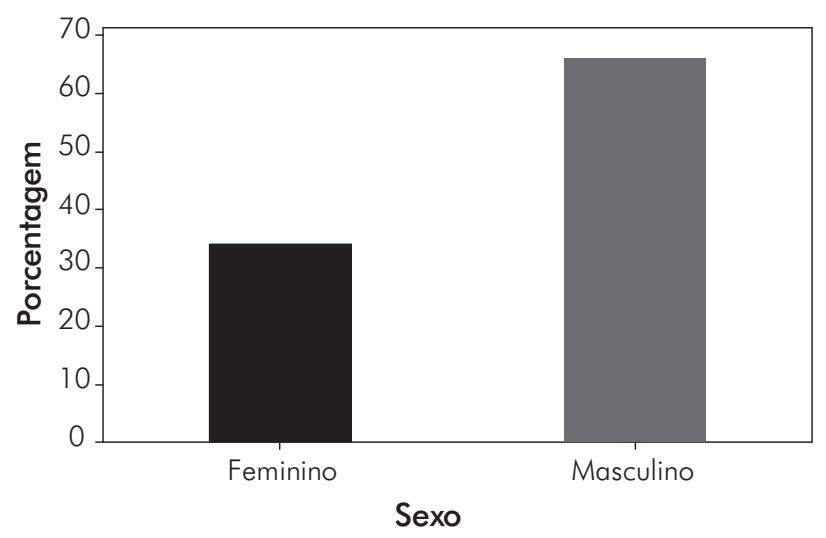

Figura 1

Distribuição dos pacientes por sexo. 
ficativamente maior (teste do $\chi^{2} \operatorname{com} p<0,001$ ), não havendo diferença significativa entre o número de acidentes com carro e com moto (teste do $\chi^{2}$ com $p=0,511$ ).

Em relação ao tipo de fratura, encontramos 37 do tipo A, 46 do tipo B e 17 do tipo C. Não houve diferença estatística entre a proporção de fraturas tipo A e tipo B (teste do $\chi^{2}$ com $p=0,323$ ) (Figura 4), mas a porcentagem de fratura tipo $\mathrm{C}$ foi significativamente menor (teste do $\chi^{2} \operatorname{com} \mathrm{p}=0,001$ ).

Quando se comparou o tipo de fratura entre os sexos, não foram encontradas diferenças significativas (teste do $\left.\chi^{2} \operatorname{com} \mathrm{p}=0,358\right)$.

Quando foi avaliado o status neurológico (Figura 5), observou-se um predomínio do Frankel E com um total de 89 pacientes, Frankel D em 4, Frankel C em 2, Frankel B em 1 e Frankel A em 4 pacientes. Analisando os dados, verificou-se que a proporção de Frankel igual a E é significativamente maior (teste do $\chi^{2} \operatorname{com} p<0,001$ ), não havendo diferença significativa entre as proporções dos outros tipos de Frankel (teste do $\chi^{2}$ com $\mathrm{p}=0,484$ ).

A junção toracolombar foi a mais acometida, sendo L1 a principal vértebra fraturada em um total de 41 fraturas acompanhada de L2 com 17 vértebras e T12 com 15 vértebras. A análise estatística mostra que a proporção de L1

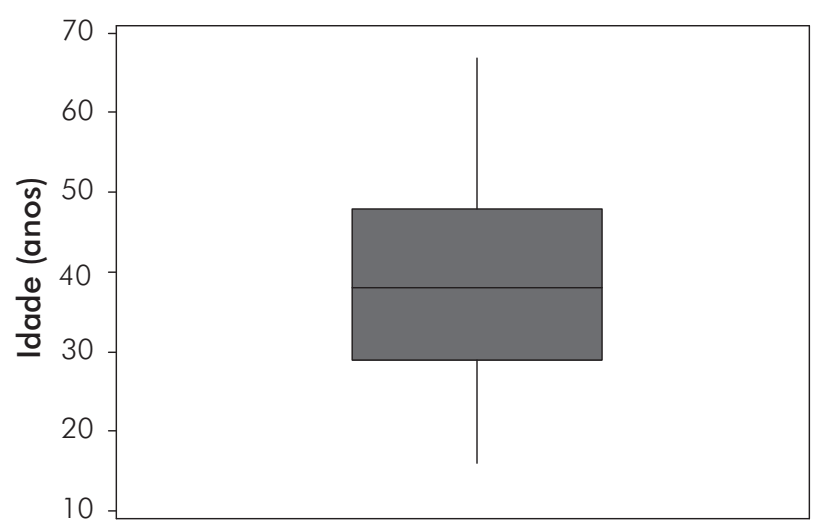

Figura 2

Média de idade dos pacientes.

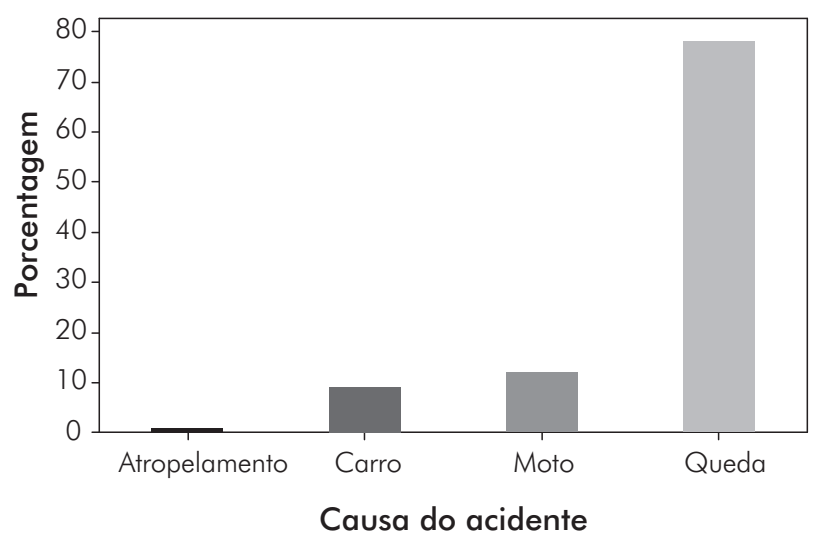

Figura 3

Distribuição por mecanismo de trauma é significativamente maior do que as demais (teste do $\chi^{2}$ com $\mathrm{p}<0,001)$. Não encontramos diferenças significativas entre L2 e T12 (teste do $\chi^{2}$ com $\mathrm{p}=0,724$ ), mas encontramos diferenças significativas entre estes dois e os demais a partir de mais de um $(p<0,025)$. Não encontramos diferenças significativas a partir de T11 com os demais que possuíam proporções menores ( $\mathrm{p}=0,101)$ (Figura 6).

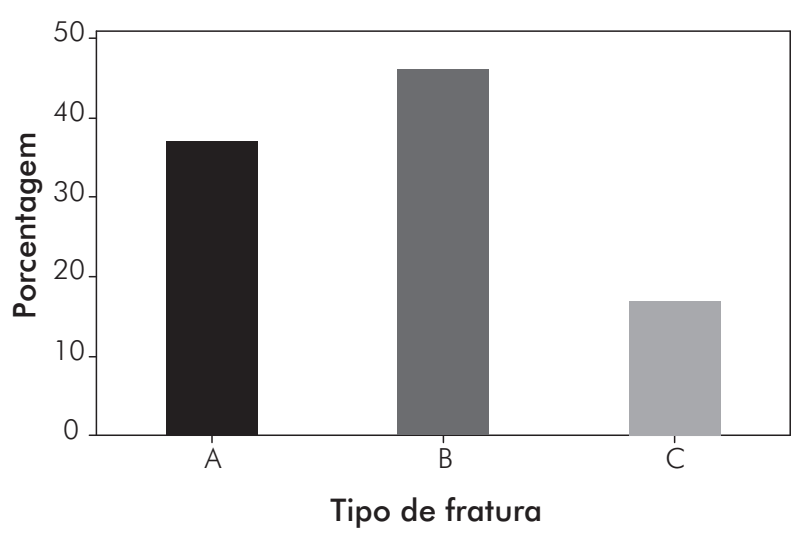

Figura 4

Relação quanto ao tipo de fratura: classificação $A O$

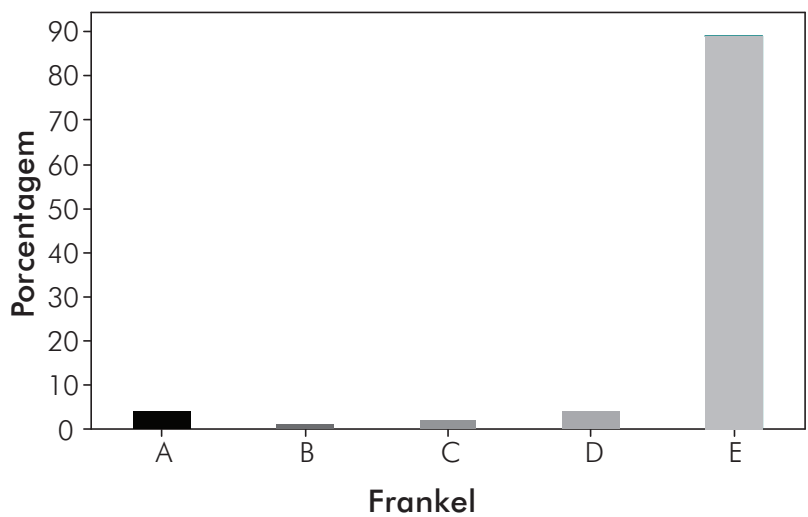

Figura 5

Déficit neurológico no momento de atendimento do paciente.

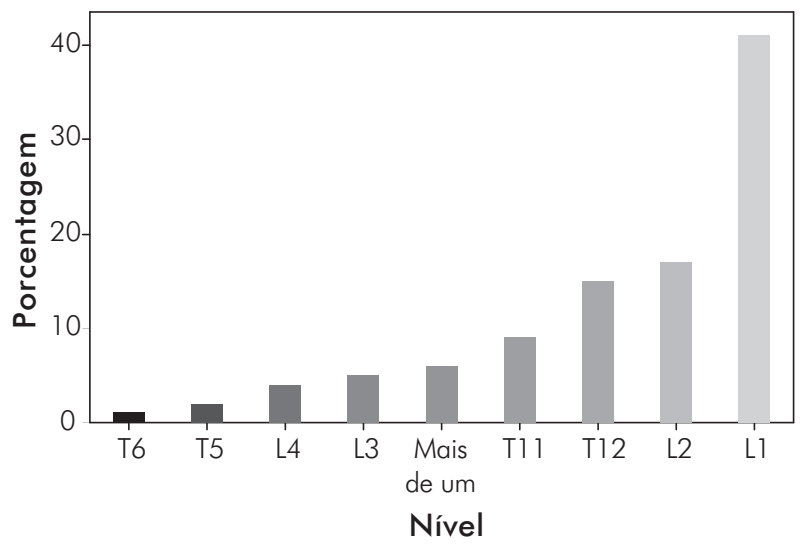

Figura 6

Relação do nível fraturado. 
Todos os pacientes foram submetidos a tratamento cirúrgico por vias posterolateral com parafusos pediculares tipo fixador interno Synthes ${ }^{\circledR}$ e artrodese com enxerto de ilíaco.

\section{Avaliação da comparação entre as variáveis}

Quando se avaliou a média de idade entre os sexos, foi observada uma média de 37,7 anos (mínimo de 17 e máximo de 67) para o sexo masculino e de 40,9 anos (mínimo de 16 e máximo de 62) para o sexo feminino. Não encontramos diferenças significativas nas distribuições de idade entre os sexos (teste de Mann-Whitney com $\mathrm{p}=0,183$ ) (Figura 7).

A causa principal do trauma não apresentou variação estatística relevante levando-se em consideração a queda de altura, mas houve diferenças significativas entre os sexos: a proporção de acidentes com motos entre pacientes do sexo masculino é significativamente maior que para o sexo feminino, enquanto a proporção de acidentes com carros no sexo masculino é significativamente menor do que no sexo feminino (teste do $\chi^{2}$ com $\mathrm{p}=0,009$ ) (Figuras 8 e 9).

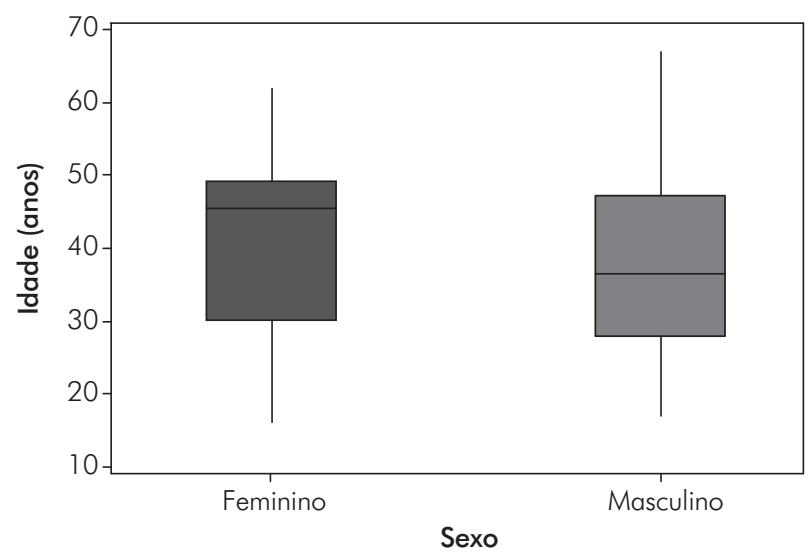

Figura 7

Relação entre média de idade e sexo.

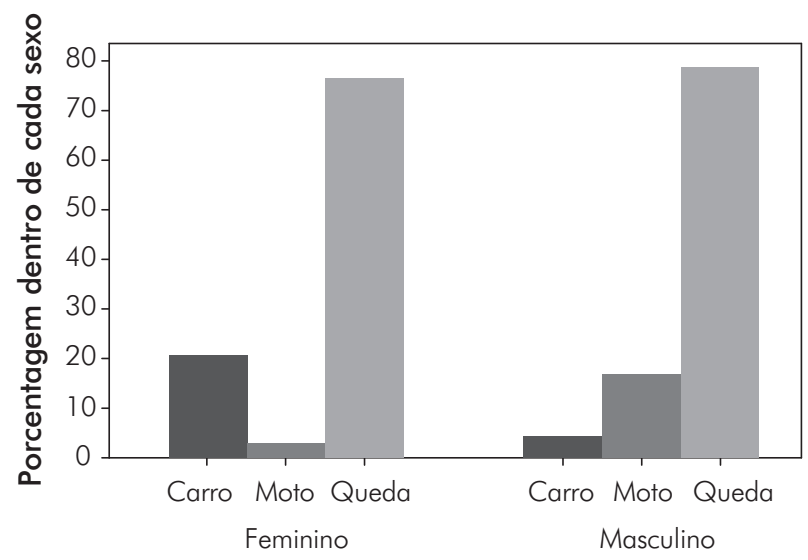

Figura 8

Correlação entre sexo, idade e mecanismo de trauma.

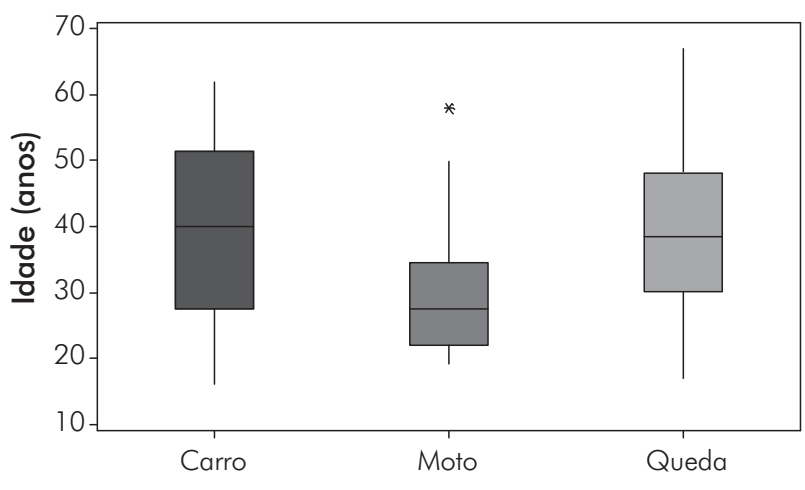

\section{Causa do acidente}

Figura 9

Correlação entre a média de idade e o mecanismo de trauma.

O tipo de fratura, quando distribuído de acordo com o sexo, não apresentou diferença significativa (teste do $\chi^{2}$ com $\mathrm{p}=0,358$ ). Quando foram avaliados sexo e déficit neurológico, também não foram encontradas diferenças (teste do $\chi^{2}$ com $\left.\mathrm{p}=0,255\right)$. Não se observou diferença entre os sexos e o local da fratura (teste do $\chi^{2} \operatorname{com} \mathrm{p}=0,664$ ).

\section{DISCUSSÃO}

Este estudo avaliou a incidência e etiologia de fraturas toracolombares em cem pacientes submetidos a tratamento cirúrgico em um único hospital terciário da Zona Leste de São Paulo. Foi observado que os pacientes mais acometidos são jovens e produtivos. Nosso trabalho concorda com outros dados referentes ao fato de as fraturas serem comuns em homens jovens ${ }^{5,6}$. Porém, foi encontrada uma média de idade um pouco acima daquela encontrada nos trabalhos apresentados, de 38 anos em comparação a 35,3 em trabalhos prévios? ${ }^{7}$. Não possuímos dados prévios, porém dados americanos indicam que a média de idade das vítimas de traumatismo vertebrais vem aumentando, como de 28,6 em 1979 para 33,5 em 19898,9.

A etiologia dos acidentes apresenta mudanças quando comparada a outros trabalhos, sendo que acidentes automobilísticos correspondem à principal causa deste tipo de fratura ${ }^{9}$, e queda representa apenas $20 \%$. Um estudo feito em Ontário afirma que quedas acidentais correspondem a quase $50 \%$ dos pacientes ${ }^{10}$, mas no presente estudo foi observado que queda corresponde a $78 \%$ dos casos, sendo queda de laje responsável por $75 \%$ dos casos e acidentes envolvendo veículos automotores a 22\%. Esta discrepância em relação à literatura pode ser relacionada com alterações culturais e mesmo habitacionais.

Estes dados mostram que a população jovem, principalmente do sexo masculino, é o principal grupo de risco para fraturas da coluna toracolombar e, apesar de existir uma diferença entre os sexos quando se correlacionou moto e carro, a queda de altura é a principal causa em ambos os sexos. 
Lesões traumáticas são a principal causa de morte e morbidades em adultos jovens ${ }^{11}$. Lenehan et al. ${ }^{12}$ corrobora nossos dados em relação ao sexo e à média de idade, relatando que homens com idade média de 40 anos são a população de maior risco. Avaliando o déficit neurológico, trabalhos apresentam uma incidência de $35 \%$ dos pacientes com algum grau de comprometimento ${ }^{13}$. Nosso trabalho apresenta um total de $11 \%$ de casos com alguma ou total alteração neurológica.

Observamos que o déficit neurológico está associado também ao grau de comprometimento da fratura. Encontramos diferenças significativas nas proporções entre os diferentes tipos de fraturas. Analisando as diferenças estabelecidas, encontramos: Frankel tipo A é significativamente maior em fratura tipo $\mathrm{C}$; Frankel tipo $\mathrm{E}$ é significativamente maior em fraturas tipo $\mathrm{A}$ e $\mathrm{B}$ do que em C; os demais tipos de Frankel não apresentaram diferenças significativas entre os diferentes tipos de fraturas.

Hu et al. ${ }^{14}$ relatam uma incidência maior de fraturas na região traço lombar, mas apresentam dificuldade de especificar qual vértebra é mais acometida, pois se trata de um trabalho retrospectivo. Na presente pesquisa também foi observada a mesma região acometida, porém registrou-se que L1 é a principal vértebra fraturada, seguida de L2 e T12.

Koch et al. ${ }^{15}$ realizaram um estudo epidemiológico no Hospital do Trabalhador no Paraná, incluindo todos os tipos de fraturas da coluna vertebral e também lesões por arma de fogo, fato não incluído em nosso estudo. Alguns dados foram semelhantes, havendo um predomínio de acidentes por queda em $51 \%$ dos casos e $32 \%$ de acidentes com veículos automotores, observando-se que os acidentes por queda foram responsáveis pelo maior número de intervenções cirúrgicas.

Pereira et al. ${ }^{16}$, em seu recente trabalho no Hospital Getúlio Vargas em Recife (PE), fez um estudo epidemiológico semelhante incluindo os mesmos segmentos, porém avaliando todas as fraturas independentemente do tratamento. Observamos que alguns dados são semelhantes à média de idade encontradas pelo autor, de 39 anos, apenas um ano a mais que o valor encontrado em nosso estudo, com grande predomínio do sexo masculino. Observamos que o mecanismo de trauma independentemente da região do país sempre se assemelha, tendo sido encontrada uma incidência de $62 \%$ dos pacientes vítimas de queda de altura e aproximadamente $23 \%$ de lesões após traumas envolvendo veículos automotores, conforme verificado em nosso estudo, assim como no artigo de Pereira et al., a vértebra mais comumente fraturada foi L1, sendo o maior índice de fraturas do tipo A, com base na Classificação AO.

Campos et al. ${ }^{17}$ fizeram uma análise epidemiológica na Zona Sul da Cidade de São Paulo, onde foram incluídos todos os segmentos da coluna vertebral sem diferenciação quanto ao tratamento; alguns dados estatísticos são semelhantes, como a prevalência no mecanismo de trauma, com $40 \%$ das fraturas ocasionadas por queda e um predomínio de $86 \%$ de indivíduos do sexo masculino associados a elevado número de quedas de lajes.

Observamos, em outro trabalho, realizado no $\mathrm{ABC}$ paulista - Hospital Mário Covas, onde Gonçalves et al. ${ }^{18}$ fizeram uma avaliação de pacientes com lesão medular, que os dados se repetem, existindo uma elevada prevalência de queda de altura, em sua grande maioria queda de lajes com um total de $47 \%$ dos casos avaliados, seguidos por acidentes automobilísticos. Observou-se também um predomínio do sexo masculino, como no presente trabalho

\section{CONCLUSÃO}

Este estudo mostra o tipo de população de risco: sexo predominantemente masculino com média de idade de 38 anos, residentes em casas da região periférica da cidade e que utilizam as lajes das residências para inúmeras atividades sem nenhuma forma de proteção tanto para os adultos como para crianças, e isto é um importante dado, pois a prevenção primária é a melhor maneira de evitar a fratura da coluna vertebral. Observou-se que a causa é multifatorial, mas que a queda de altura, principalmente de laje, corresponde à grande maioria dos casos, que os pacientes vítimas de trauma são jovens e produtivos, e que este tipo de trauma é frequente nas mais diversas regiões do Brasil.

Sugere-se que mudanças, principalmente na área habitacional, com leis que regulamentem as lajes, e uma política de fiscalização possam, de fato, reduzir o número de acidentes e, consequentemente, os gastos em internações, cirurgias e reabilitação, além de perdas com parada da produção por estes pacientes e gastos com previdência.

\section{REFERÊNCIAS}

1. Zona Leste de São Paulo. Wikipédia enciclopédia livre [Internet]. [citado 2010 Jan 13] Disponível em: http:// pt.wikipedia.org/wiki/Zona_leste_de_ São_Paulo\#Refer.C3.AAncias.

2. De Vivo MJ. Causes and costs of spinal injury in the United States. Spinal Cord. 1977;35(12):809-13.

3. Magerl F, Aebi M, Harms J, Nazarian S. A comprehensive classification of thoracic and lumbar injuries. Eur Spine J. 1994;3(4):184-201.
4. Frankel HL, Hancock DO, Hyslop G. The value of postural reduction in the initial management of closed injuries of the spine with paraplegia and tetraplegia. Paraplegia. 1969;7(3):179.

5. Surkin J, Gilbert BJC, Harkey HL 3rd, Sniezek J, Currier M. Spinal cord injury in Mississippi: Findings and evaluation, 1992-1994. Spine. 2000;25(6):716-21.
6. Thurman DJ, Burnett CL, Jeppson L, Beaudoin DE, Sniezek JE. Surveillance of spinal cord injuries in Utah, USA. Paraplegia. 1994;32(10):665-9.

7. Sekhon LH, Fehlings MG. Epidemiology, demographics, and pathophysiology of acute spinal cord injury. Spine. 2001;26(24 Suppl):S2-12. 
8. Burney RE, Maio RF, Maynard F, Karunas R. Incidence, characteristics, and outcome of spinal cord injury at trauma centers in North America. Arch Surg. 1993;128(5):596-9.

9. Pickett GE, Campos-Benitez M, Keller JL, Duggal N. Epidemiology of traumatic spinal cord injury in Canada. Spine. 2006;31(7):799-805.

10.Pickett W, Simpson K, Walker J, Brison R. Traumatic spinal cord injury in Ontario, Canada. J Trauma. 2003;55(6):1070-6.

11.WHO. Statistical Information System (WHOSIS). [Internet]. WHO; 2007. [cited 2010 Jun 12]. Available from: http://www.who.int/whosis/en/

12.Lenehan B, Boran S, Street J, Higgins T, McCormack D, Poynton AR. Demographics of acute admissions to a National Spinal Injuries Unit. Eur Spine J. 2009;18(7):938-42.

13. Wyndaele M, Wyndale J-J. Incidence, prevalence and epidemiology of spinal cord injury: what learns a worldwide literature survey? Spinal Cord. 2006;44(9):523-9
14.Hu R, Mustard CA, Burns C. Epidemiology of incident spinal fracture in a complete population. Spine. 1996;21(4):492-9.

15.Koch A, Graells XS, Zaninelli EM. Epidemiologia de fraturas da coluna de acordo com o mecanismo de trauma: análise de 502 casos. Coluna/ Columna. 2007;6(1):18-23.

16.Pereira AFF, Portela LED, Lima GDA, Carneiro WCG, Ferreira MAC, Rangel TAM, et al. Avaliação epidemiológica das fraturas da coluna torácica e lombar dos pacientes atendidos no serviço de Ortopedia e Traumatologia do Hospital Getúlio Vargas em Recipe/ PE. Coluna/Columna. 2009;8(4): 395-400

17.Campos MF, Ribeiro AT, Listik S, Pereira CAB, Andrade Sobrinho J, Rapoport A. Epidemiologia do traumatismo da coluna vertebral. Rev Col Bras Cir. 2008;35(2):88-93.
18.Gonçalves AMT, Rosa LN, D’Angelo CT, Savordelli CL, Bonin GL, Squarcino IM, et al. Aspectos epidemiológicos da lesão medular na área de referência do Hospital Estadual Mário Covas. Arq Med ABC. 2007;32(2):64-6.

\section{Correspondência}

Luiz Claudio Lacerda Rodrigues

Setor de Ortopedia e Traumatologia do Hospital Santa Marcelina

Rua Santa Marcelina, 177 - Itaquera

CEP: 08270-070 - São Paulo (SP), Brasil

Fone: (1 1) 2170-6000, ramal 6317

E-mail: luizclr@terra.com.br 